\title{
Semiclassical Theory for Many-Body Fermionic Systems
}

\author{
Pierre Gaspard and Sudhir R. Jain \\ Faculté des Sciences and \\ Center for Nonlinear Phenomena and Complex Systems, \\ Université Libre de Bruxelles, Campus Plaine C.P. 231, \\ Boulevard du Triomphe, 1050 Bruxelles, Belgium
}

\begin{abstract}
We present a treatment of many-body Fermionic systems that facilitates an expression of the well-known quantities in a series expansion in $\hbar$. The ensuing semiclassical result contains to a leading order of the response function the classical time correlation function of the observable followed by the WeylWigner series, on top of these terms are the periodic-orbit correction terms. The treatment given here starts from linear response assumption of the manybody theory and in its connection with semiclassical theory, it makes no assumption of the integrability of classical dynamics underlying the one-body quantal system. Applications of the framework are also discussed.
\end{abstract}

PACS numbers:05.30.-d 05.45.+b 


\section{Introduction}

Semiclassical framework for single-particle systems is presently in an advanced stage [1.2]. For both the integrable and chaotic dynamics, we now understand the semiclassical quantization. However, for intermediate [3] and mixed dynamical scenario, we still lack a convincing theory. Another situation of a great practical relevance arises in the systems comprising of many bodies. That the spectral fluctuation characteristics of these systems are modelled in much the same way as for chaotic systems with lesser degrees of freedom has been shown quite recently [4]. It is very important to note that complex systems do possess features, mainly associated with the generic nature of the thermodynamic limit, which are completely absent from the systems with fewer freedoms, friction being one of the examples. There are other systems which one would like to understand semiclassically. Some notable examples are of quantum dots [5], metallic clusters [6] and their optical properties [7]; also, nuclear physics at high spins [8] presents us with opportunity to test various semiclassical ideas in many-body theory. There has been an attempt based on the semiclassical limit of the time-dependent Hartree-Fock equation where one can establish a connection [9] between the strength function given by the Vlasov equation and the corresponding quantum function in the limit of large quantum numbers. Analogous to the many-body treatment based on random-phase approximation (RPA), the linearized Vlasov equation gives an integral equation for the particle-hole propagator in terms of classical propagator in the static mean field and two-particle Coulomb interaction. Thus one needs to evaluate the classical propagator in the mean field and then solve the integral equation.

There is a lot of evidence that periodic orbits in the semiclassical trace formulae describe the shell effects in metallic clusters [10]. Of course, these have been preceded by nuclear physicists [11] by more than a decade. In fact, the periodic-orbit theory [12], which, in principle, should be rendered useless for practical purposes due to a huge number of technical problems associated with finding periodic orbits and summing a conditionally convergent series, gets encouragement from observations that only a few periodic orbits are enough in many situations [12]. 
We present here a semiclassical treatment of the response function. Response function is essentially the imaginary part of generalized dynamical susceptibility which, in turn, is related to the correlation commutator. In the section 2, we present the general discussion from the linear response theory to arrive at a quantity for which semiclassical expression can be written. The key point of this section is to show that the most important quantity is a time correlation function. In the section 3, we present semiclassical treatment of the system perturbed by an external influence. This enables us to write the relevant results of sections 2 and 3 in a semiclassical expansion. We will see that the leading term is indeed the two-time correlation function averaged over the phase space, followed by the Weyl-Wigner series which has the periodic-orbit corrections. On our way, we take account of the Fermionic nature of the particles.

\section{Response Function and the Time Correlation Function}

We present here a discussion on the response of a system in the presence of an external field. We work with susceptibility [13] and express it in terms of trace over single-particle states of two-time correlation function of the observable. This quantity is also known as the polarization propagator [14].

If a system described by the Hamiltonian $\hat{H}$ is externally disturbed by an field $F^{e x t}(t)$, then the total Hamiltonian is

$$
\hat{H}_{T}=\hat{H}-\hat{Q} F^{e x t}(t)
$$

where $\hat{Q}$ is an observable, an example could be magnetization in the context of spin systems, or, an electric dipole operator in an example involving photoabsorption, and so on. The response function can be written as the imaginary part of the dynamical susceptibility,

$$
\begin{aligned}
\chi^{\prime \prime}\left(t, t^{\prime}\right) & =(2 \hbar)^{-1}\left\langle\left[\hat{Q}(t), \hat{Q}\left(t^{\prime}\right)\right]\right\rangle \\
& =\int \frac{d \omega}{2 \pi} e^{-i \omega\left(t-t^{\prime}\right)} \tilde{\chi}^{\prime \prime}(\omega) .
\end{aligned}
$$

The angular brackets denote the expectation value and the square brackets denote the commutator. Setting an initial time to 0 and the final time to $t$, we can write 


$$
\chi^{\prime \prime}(t)=(2 \hbar)^{-1}[\langle\hat{Q}(t) \hat{Q}(0)\rangle-\langle\hat{Q}(0) \hat{Q}(t)\rangle]
$$

where

$$
\hat{Q}(t)=\exp (i \hat{H} t / \hbar) \hat{Q} \exp (-i \hat{H} t / \hbar)
$$

and where $\langle\cdot\rangle$ denotes the average over the initial state of the system which is, for instance, the thermal state wherein

$$
\langle\cdot\rangle=\frac{1}{Z(\beta)} \operatorname{tr} e^{-\beta \hat{H}}(\cdot)
$$

with

$$
Z(\beta)=\operatorname{tr} \exp (-\beta \hat{H})
$$

if the system was in contact with a thermal reservoir at temperature $T=1 / \beta k_{B}$ in the period preceding the interaction with the external field, $F^{e x t}(t)$. If the system was in its pure ground state $\left|\Phi_{0}\right\rangle$ then the average is over this many-body eigenstate of $\hat{H}$, i.e.,

$$
\langle\cdot\rangle=\left\langle\Phi_{0}|\cdot| \Phi_{0}\right\rangle .
$$

In this case, we can write $\chi^{\prime \prime}(t)$ as

$$
\begin{aligned}
\chi^{\prime \prime}(t) & =(2 \hbar)^{-1}\left[\left\langle\Phi_{0}|\hat{Q}(t) \hat{Q}(0)| \Phi_{0}\right\rangle-\left\langle\Phi_{0}|\hat{Q}(0) \hat{Q}(t)| \Phi_{0}\right\rangle\right] \\
& =(2 \hbar)^{-1}\left[e^{i E_{0} t / \hbar}\left\langle\Phi_{0}\left|\hat{Q} e^{-i \hat{H} t / \hbar} \hat{Q}\right| \Phi_{0}\right\rangle-e^{-i E_{0} t / \hbar}\left\langle\Phi_{0}\left|\hat{Q} e^{i \hat{H} t / \hbar} \hat{Q}\right| \Phi_{0}\right\rangle\right] \\
& =(2 \hbar)^{-1} \sum_{n}\left[e^{i E_{0} t / \hbar}\left|\left\langle\Phi_{0}|\hat{Q}| \Phi_{n}\right\rangle\right|^{2} e^{-i E_{n} t / \hbar}-e^{-i E_{0} t / \hbar}\left|\left\langle\Phi_{0}|\hat{Q}| \Phi_{n}\right\rangle\right|^{2} e^{i E_{n} t / \hbar}\right]
\end{aligned}
$$

where $\left\{\left|\Phi_{n}\right\rangle\right\}$ denote all the many-body eigenstates of the isolated-system Hamiltonian,

$$
\hat{H}\left|\Phi_{n}\right\rangle=E_{n}\left|\Phi_{n}\right\rangle,
$$

with $E_{n} \geq E_{0}$.

Upon Fourier transformation,

$$
\begin{aligned}
\tilde{\chi}^{\prime \prime}(\omega) & =\pi \sum_{n}\left|\left\langle\Phi_{0}|\hat{Q}| \Phi_{n}\right\rangle\right|^{2}\left[\delta\left(\hbar \omega+E_{0}-E_{n}\right)-\delta\left(\hbar \omega-E_{0}+E_{n}\right)\right] \\
& =\sum_{n}\left|\left\langle\Phi_{0}|\hat{Q}| \Phi_{n}\right\rangle\right|^{2} \Im\left(\frac{1}{\hbar \omega-E_{0}+E_{n}+i 0^{+}}-\frac{1}{\hbar \omega+E_{0}-E_{n}+i 0^{+}}\right)
\end{aligned}
$$


where we used the identity

$$
\frac{1}{x+i 0^{+}}=\mathcal{P}\left(\frac{1}{x}\right)-i \pi \delta(x)
$$

$\mathcal{P}$ denoting the Cauchy principal value. Methods have been developed to evaluate semiclassically such expressions as above [1]. However, we should recall that the system is many-body so that such methods would require the search for classical orbits of the many-body system. Simplification arises by taking account of the Fermionic character of the system which allows a reduction of the problem to one-body Hamiltonian in an effective potential determined by, for instance, the Hartree-Fock method. We shall make this simplifying assumption here and restrict our system to a set of uncoupled one-body Hamiltonians. A similar assumption is carried out on the coupling operator for which we assume the same form as for the Hamiltonian,

$$
\begin{aligned}
& \hat{H}=\sum_{i=1}^{N} \hat{h}_{i}, \\
& \hat{Q}=\sum_{i=1}^{N} \hat{q}_{i}
\end{aligned}
$$

where $\hat{h}_{i}$ and $\hat{q}_{i}$ are one-body operators. Let us denote the one-body eigenstates as

$$
\hat{h}\left|\phi_{a}\right\rangle=\epsilon_{a}\left|\phi_{a}\right\rangle
$$

When the Fermionic many-body system is in its ground state, all the one-body eigenstates are occupied up to the Fermi energy, $\epsilon_{F}$ so that the energy of the ground state is

$$
E_{0}=\sum_{\substack{a=1 \\ \epsilon_{a}\left\langle\epsilon_{F}\right.}}^{N} \epsilon_{a} .
$$

In any many-body excited state, $\left|\Phi_{n}\right\rangle$, at least one 1-body level above the Fermi energy is occupied. We may denote such excited states by the list of the occupied 1-body levels, or equivalently, by the list of the one-body levels for which the occupation is different with respect to the ground state $\left|\Phi_{0}\right\rangle$, having in mind that such states are antisymmetric for an exchange of two Fermions: 


$$
\begin{aligned}
& \left|\Phi_{0}\right\rangle=\left|1111 \ldots 11 \vdots^{\epsilon_{F}} 00 \ldots 0\right\rangle \\
& \left|\Phi_{n}\right\rangle=\left|111 \ldots 1110 \vdots^{\epsilon_{F}} 0100 \ldots\right\rangle .
\end{aligned}
$$

For operators which are sums of 1-body operators as assumed in (12), we obtain the result that the matrix elements $\left\langle\Phi_{0}|\hat{Q}| \Phi_{n}\right\rangle$ are non-vanishing only for the states $\Phi_{n}$ which differ from the ground state $\Phi_{0}$ by one excitation. These states have a hole in state $a\left(\epsilon_{a}\left\langle\epsilon_{F}\right)\right.$ and a particle in state $\left.b\left(\epsilon_{b}\right\rangle \epsilon_{F}\right)$. For these states the matrix elements are thus

$$
\left\langle\Phi_{0}|\hat{Q}| \Phi_{n}\right\rangle=\left\langle\phi_{a}|\hat{q}| \phi_{b}\right\rangle
$$

Moreover the energy of the excited state is

$$
E_{n}=E_{0}-\epsilon_{a}+\epsilon_{b}
$$

under the assumption that $\epsilon_{a}\left\langle\epsilon_{F}\left\langle\epsilon_{b}\right.\right.$. After some standard manipulations, we can write

$$
\begin{aligned}
& \sum_{n} \frac{\left|\left\langle\Phi_{0}|\hat{Q}| \Phi_{n}\right\rangle\right|^{2}}{\hbar \omega-E_{0}+E_{n}+i 0^{+}} \\
= & \sum_{a, b} \frac{\left|\left\langle\phi_{a}|\hat{q}| \phi_{b}\right\rangle\right|^{2}}{\hbar \omega-\epsilon_{a}+\epsilon_{b}+i 0^{+}}\left[1-\Theta\left(\epsilon_{F}-\epsilon_{b}\right)\right] \Theta\left(\epsilon_{F}-\epsilon_{a}\right),
\end{aligned}
$$

where the Heaviside step function, $\Theta(\cdot)$ takes care of the aforementioned restriction on the location of the 1-body states $\phi_{a}$ and $\phi_{b}$ with respect to $\epsilon_{F}$.

In the presence of a thermal reservoir at temperature $T\left[=1 /\left(\beta k_{B}\right)\right]$ described with canonical density matrix, we get that

$$
\begin{aligned}
\langle\hat{Q}(t) \hat{Q}(0)\rangle & =\frac{1}{Z} \operatorname{tr} e^{-\beta \hat{H}} e^{i t \hat{H} / \hbar} \hat{Q} e^{-i t \hat{H} / \hbar} \hat{Q} \\
& =\sum_{m n} \exp \left[i E_{m}(t+i \beta \hbar) / \hbar\right] \exp \left(-i E_{n} t / \hbar\right)\left|\left\langle\Phi_{m}|\hat{Q}| \Phi_{n}\right\rangle\right|^{2} .
\end{aligned}
$$

Thus the dynamical susceptibility is

$$
\tilde{\chi} "(\omega)=\frac{1}{Z(\beta)} \sum_{m n}\left|\left\langle\Phi_{m}|\hat{Q}| \Phi_{n}\right\rangle\right|^{2} \Im \frac{\exp \left(-\beta E_{m}\right)}{\hbar \omega-E_{m}+E_{n}+i 0^{+}}-(\omega \rightarrow-\omega) .
$$

Similar assumptions as before enable us to reduce this expression to the 1-body system. Now the probability to find a state $\left|\Phi_{m}\right\rangle$ in which $\left|\phi_{a}\right\rangle$ is occupied is given by the FermiDirac distribution at energy $\epsilon_{a}$. But $\left|\Phi_{n}\right\rangle$ is related to $\left|\Phi_{m}\right\rangle$ by the fact that $\left|\phi_{b}\right\rangle$ must be 
unoccupied (see above). So, we have a joint probability that $\left|\phi_{a}\right\rangle$ is occupied and $\left|\phi_{b}\right\rangle$ is unoccupied, hence the propagator is

$$
\tilde{\chi}^{\prime \prime}(\omega)=\sum_{a, b}\left|\left\langle\phi_{a}|\hat{q}| \phi_{b}\right\rangle\right|^{2} \Im \frac{p_{a}^{F D}-p_{b}^{F D}}{\hbar \omega-\epsilon_{a}+\epsilon_{b}+i 0^{+}}
$$

where

$$
p_{a}^{F D}=\frac{1}{\exp \left[\beta\left(\epsilon_{a}-\mu\right)\right]+1}
$$

denotes the Fermi-Dirac probability or mean occupation number. $\mu$ is the chemical potential fixed by the total number of Fermions in the system. Eq. (21) gives therefore the Fourier transform of the two-time correlation function.

At zero temperature, the Fermi-Dirac distribution becomes a Heaviside step function, so the expression (21) becomes

$$
\tilde{\chi}^{\prime \prime}(\omega)=\sum_{a, b}\left|\left\langle\phi_{a}|\hat{q}| \phi_{b}\right\rangle\right|^{2} \Im \frac{\Theta\left(\epsilon_{F}-\epsilon_{a}\right)-\Theta\left(\epsilon_{F}-\epsilon_{b}\right)}{\hbar \omega-\epsilon_{a}+\epsilon_{b}+i 0^{+}}
$$

which is identical with the expression previously derived. In the foregoing discussion of this section, we have started from the time correlation function (actually the commutator) and written an expression for the response. Now that the susceptibility has been reduced to a 1-body expression, we can treat the 1-body system semiclassically in terms of periodic orbits in the effective Hartree-Fock Hamiltonian $\hat{h}$.

It is well to recall that $\tilde{\chi}^{\prime \prime}(\omega)$ has a physical interpretation in terms of energy dissipation which is related to the fluctuations in the frequency spectrum obtained as the Fourier transform of the time correlation function. Indeed the fluctuation-dissipation theorem [13] lies just in realizing that connection.

Employing the identity (11) in (21), and expanding the difference between $p^{F D}$ 's in the resulting expression, we obtain

$$
\begin{aligned}
\tilde{\chi}^{\prime \prime}(\omega) & =\pi \sum_{a, b}\left|\left\langle\phi_{a}|\hat{q}| \phi_{b}\right\rangle\right|^{2}\left[p^{F D}\left(\epsilon_{a}\right)-p^{F D}\left(\epsilon_{a}+\hbar \omega\right)\right] \delta\left(\hbar \omega+\epsilon_{a}-\epsilon_{b}\right) \\
& =-\pi \sum_{n=1}^{\infty} \frac{(\hbar \omega)^{n}}{n !} \sum_{a, b} \frac{\partial^{n} p^{F D}}{\partial \epsilon^{n}}\left(\epsilon_{a}\right)\left|\left\langle\phi_{a}|\hat{q}| \phi_{b}\right\rangle\right|^{2} \delta\left(\hbar \omega+\epsilon_{a}-\epsilon_{b}\right) \\
& =-\frac{1}{2 \hbar} \sum_{n=1}^{\infty} \frac{(\hbar \omega)^{n}}{n !} \tilde{f}_{n}(\omega) .
\end{aligned}
$$


where we introduced the expression

$$
\tilde{f}_{n}(\omega)=2 \pi \hbar \sum_{a, b} \frac{\partial^{n} p^{F D}}{\partial \epsilon^{n}}\left(\epsilon_{a}\right)\left|\left\langle\phi_{a}|\hat{q}| \phi_{b}\right\rangle\right|^{2} \delta\left(\hbar \omega+\epsilon_{a}-\epsilon_{b}\right)=\int d t e^{i \omega t} f_{n}(t),
$$

which is the Fourier transform of the time correlation functions

$$
f_{n}(t)=\operatorname{tr} \frac{\partial^{n} p^{F D}}{\partial \epsilon^{n}}(\hat{h}) e^{i t \hat{h} / \hbar} \hat{q} e^{-i t \hat{h} / \hbar} \hat{q}
$$

Moreover, we can rewrite these time correlation functions in terms of a single time correlation function as follows

$$
f_{n}(t)=\int d \epsilon \frac{\partial^{n} p^{F D}}{\partial \epsilon^{n}}(\epsilon) C_{\epsilon}(t)
$$

with

$$
C_{\epsilon}(t)=\operatorname{tr} \delta(\epsilon-\hat{h}) \hat{q}(t) \hat{q}(0) \quad \text { and } \quad \hat{q}(t)=e^{i t \hat{h} / \hbar} \hat{q} e^{-i t \hat{h} / \hbar}
$$

In summary, we have so far reduced the many-body dynamical susceptibility to an expression involving the time correlation function $C_{\epsilon}(t)$ of the 1-body effective dynamics:

$$
\tilde{\chi}^{\prime \prime}(\omega)=-\pi \sum_{n=1}^{\infty} \frac{(\hbar \omega)^{n}}{n !} \int \frac{d t d \epsilon}{2 \pi \hbar} e^{i \omega t} \frac{\partial^{n} p^{F D}}{\partial \epsilon^{n}}(\epsilon) C_{\epsilon}(t) .
$$

We will see in the next section that this expression is particularly suited for developing semiclassical expansions.

\section{Semiclassical Expressions of Response}

According to the preceding section, we need to write the semiclassical expression for the time correlation function $C_{\epsilon}(t)$ in order to obtain semiclassically the response function. To this end, we consider the correlation function in the form:

$$
C_{\epsilon}(t)=\operatorname{tr} \delta(\epsilon-\hat{h}) \hat{X}=\operatorname{tr} \delta(\epsilon-\hat{h}) \hat{A}+i \operatorname{tr} \delta(\epsilon-\hat{h}) \hat{B}
$$

where $\hat{X}=\hat{X}(t)=\hat{q}(t) \hat{q}(0)$ is a 1-body operator. This operator is nonHermitian but can be decomposed as $\hat{X}=\hat{A}+i \hat{B}$ in terms of two Hermitian operators: $\hat{A}=\left(\hat{X}+\hat{X}^{\dagger}\right) / 2$ and $\hat{B}=\left(\hat{X}-\hat{X}^{\dagger}\right) /(2 i)$. 
Methods have been obtained to evaluate semiclassically such expressions in terms of periodic orbits [1,15]. To motivate this method, we begin by observing [16] that such an expression involves the matrix elements of $\hat{A}$ (and $\hat{B}$ ) over the eigenstates of $\hat{h}$. These matrix elements can be obtained at the first-order perturbation theory of a perturbed Hamiltonian $\hat{h}(\lambda)=\hat{h}+\lambda \hat{A}$. Assuming the eigenvalue problem for $\hat{h}(\lambda)$ to be solved, the matrix elements of $\hat{A}$ may thus be obtained in terms of derivatives of eigenvalues of the perturbed Hamiltonian, $\epsilon_{n}(\lambda)$, with respect to $\lambda$. Now the periodic-orbit theory by Gutzwiller can be used to calculate the diagonal matrix elements.

Each term of the correlation function $C_{\epsilon}(t)$ can therefore be expressed as

$$
\begin{aligned}
\operatorname{tr} \delta(\epsilon-\hat{h}) \hat{A} & =-\frac{1}{\pi} \Im \operatorname{tr} \frac{\hat{A}}{\epsilon-\hat{h}+i 0^{+}} \\
& =\left.\frac{1}{\pi} \Im \operatorname{tr} \frac{\partial}{\partial \lambda} \log \left(\epsilon-\hat{h}-\lambda \hat{A}+i 0^{+}\right)\right|_{\lambda=0}
\end{aligned}
$$

Comparing with the following indentity

$$
\begin{aligned}
-\frac{1}{\pi} \frac{\partial}{\partial \epsilon} \Im \operatorname{tr} \log \left(\epsilon-\hat{h}-\lambda \hat{A}+i 0^{+}\right) & =\operatorname{tr} \delta(\epsilon-\hat{h}-\lambda \hat{A}) \\
& =\frac{\partial}{\partial \epsilon} N(\epsilon ; \lambda)
\end{aligned}
$$

which is the derivative of the staircase function $N(\epsilon \lambda)$ with respect to the energy, we arrive at the relation,

$$
\operatorname{tr} \delta(\epsilon-\hat{h}) \hat{A}=-\left.\frac{\partial N(\epsilon ; \lambda)}{\partial \lambda}\right|_{\lambda=0}
$$

which gives each term of the correlation function as the derivative of the staircase function of the perturbed Hamiltonian $\hat{h}(\lambda)$ with respect to $\lambda$.

On the other hand, one has the well-known semiclassical expression:

$$
\begin{aligned}
N(\epsilon ; \lambda) & =\int \frac{d^{f} x d^{f} p}{(2 \pi \hbar)^{f}} \Theta\left[\epsilon-h_{W}(\lambda)\right]+O\left(\hbar^{-f+1}\right) \\
& +\sum_{p} \sum_{r=1}^{\infty} \frac{1}{r \pi} \frac{\sin \left[\frac{r}{\hbar} S_{p}(\epsilon ; \lambda)-r \frac{\pi}{2} \nu_{p}\right]}{\left|\operatorname{det}\left[\mathbf{m}_{p}^{r}(\lambda)-\mathbf{l}\right]\right|^{1 / 2}}+O(\hbar),
\end{aligned}
$$

where $\mathbf{m}_{p}(\lambda)$ is the monodromy matrix governing the stability of the classical periodic trajectory, $p ; \nu_{p}$ is the Maslov index of the trajectory and $h_{W}$ is the Weyl-Wigner transform 
of the Hamiltonian $\hat{h}(\lambda)=\hat{h}+\lambda \hat{A}$. We notice that the above periodic orbits $p$ are those of the perturbed Hamiltonian. We assume here that the periodic orbits of $h_{W}(\lambda)$ deform continuously to the periodic orbits of $h_{W}(\lambda=0)$ for $\lambda$ small enough. For the purpose of differentiating this expression with respect to $\lambda$, we use the following classical formula which gives the derivative of the action of the periodic orbits [19],

$$
\frac{\partial S_{p}}{\partial \lambda}=-\oint d t \frac{\partial h_{W}(\lambda)}{\partial \lambda}=-\oint d t A_{W}
$$

where the integrals go around the periodic orbit, $p$, and $A_{W}$ is the Weyl-Wigner transform of the operator $\hat{A}$. Taking the derivative with respect to $\lambda$ and adding both terms composing the correlation function, we finally obtain

$$
\begin{aligned}
\operatorname{tr} \delta(\epsilon-\hat{h}) \hat{X} & =\int \frac{d^{f} x d^{f} p}{(2 \pi \hbar)^{f}} X_{W} \delta\left(\epsilon-h_{c l}\right)+O\left(\hbar^{-f+1}\right) \\
& +\frac{1}{\pi \hbar} \sum_{p} \sum_{r=1}^{\infty} \frac{\cos \left(\frac{r}{\hbar} S_{p}-r \frac{\pi}{2} \nu_{p}\right)}{\left|\operatorname{det}\left(\mathbf{m}_{p}^{r}-\mathbf{I}\right)\right|^{1 / 2}} \oint_{p} d t X_{W}+O\left(\hbar^{0}\right)
\end{aligned}
$$

where $h_{c l}=h_{W}(\lambda=0)$ and the periodic orbits are those of $h_{c l}$.

For the case like ours, when

$$
\hat{X}=\exp \left(\frac{i}{\hbar} \hat{h} t\right) \hat{q} \exp \left(-\frac{i}{\hbar} \hat{h} t\right) \hat{q}
$$

the Weyl-Wigner tranform can be written as

$$
\begin{aligned}
X_{W}(\mathbf{x}, \mathbf{p}) & =\left(e^{\frac{i}{\hbar} \hat{h} t}\right)_{W} e^{\frac{i}{2} \hbar \hat{\Lambda}}\left\{q_{W} e^{\frac{i}{2} \hbar \hat{\Lambda}}\left[\left(e^{\frac{-i}{\hbar} \hat{h} t}\right)_{W} e^{\frac{i}{2} \hbar \hat{\Lambda}} q_{W}\right]\right\} \\
& =\left[\exp \left(-\hat{\mathcal{L}}_{c l} t\right) q_{W}\right] q_{W}+(\text { Weyl corrections }) \\
& =q_{W}(t) q_{W}(0)+(\text { Weyl corrections })
\end{aligned}
$$

where $\hat{\mathcal{L}}_{c l}=\{h, \cdot\}$ is the classical evolution operator, and the operator $\hat{\Lambda}$ is standard [20] in the Weyl-Wigner expansions defined as

$$
\hat{\Lambda}=\frac{\overleftarrow{\partial}}{\partial \mathbf{p}} \cdot \frac{\vec{\partial}}{\partial \mathbf{x}}-\frac{\overleftarrow{\partial}}{\partial \mathbf{x}} \cdot \frac{\vec{\partial}}{\partial \mathbf{p}}
$$

and $q_{W}(t)=q_{W}\left[\Phi^{t}(\mathbf{x}, \mathbf{p})\right]$ is the value of $q_{W}$ at the current point $\Phi^{t}(\mathbf{x}, \mathbf{p})$ of the trajectory from the initial condition $(\mathbf{x}, \mathbf{p})$ of the Hamiltonian flow $\Phi^{t}$ of $h_{c l}$. 
Putting all the pieces together, we can now write the semiclassical expansion for the correlation function as

$$
\begin{aligned}
C_{\epsilon}(t) & =\operatorname{tr} \delta(\epsilon-\hat{h}) \hat{q}(t) \hat{q}(0) \\
& =\int \frac{d^{f} x d^{f} p}{(2 \pi \hbar)^{f}} \delta\left[\epsilon-h_{c l}(\mathbf{x}, \mathbf{p})\right] q_{W}(\mathbf{x}, \mathbf{p})\left(e^{-\hat{\mathcal{L}}_{c l} t} q_{W}\right)(\mathbf{x}, \mathbf{p})+(\text { Weyl corrections }) \\
& +\frac{1}{\pi \hbar} \sum_{p, r} \frac{\cos \left(\frac{r}{\hbar} S_{p}-r \frac{\pi}{2} \nu_{p}\right)}{\left|\operatorname{det}\left(\mathbf{m}_{p}^{r}-\mathbf{I}\right)\right|^{1 / 2}} \oint_{p} d \tau q_{W}(\tau) q_{W}(\tau+t)+O\left(\hbar^{0}\right) .
\end{aligned}
$$

We now go back to Eq. (29) where we see that we still need to evaluate the Fourier transform of the correlation function $C_{\epsilon}(t)$.

Let us evaluate the first term with $n=1$ of the susceptibility. This should give the dominant term of the Weyl series in powers of the Planck constant $\hbar$ as well as a sum over periodic orbits with the smallest power in $\hbar$ which gives therefore the most important contribution. The other periodic-orbit sums would involve smaller amplitudes with higher powers in $\hbar$. Replacing with the previous semiclassical result, we get

$$
\begin{aligned}
\tilde{\chi}^{\prime}(\omega) & =-\frac{\omega}{2} \int d \epsilon \frac{\partial p^{F D}}{\partial \epsilon}(\epsilon) \int d t e^{i \omega t} C_{\epsilon}(t)+\mathcal{O}(\hbar) \\
& =-\frac{\omega}{2} \int d t \int \frac{d^{f} x d^{f} p}{(2 \pi \hbar)^{f}} \partial_{\epsilon} p^{F D}\left[h_{c l}(\mathbf{x}, \mathbf{p})\right] q_{W}(\mathbf{x}, \mathbf{p})\left[e^{\left(i \omega-\hat{\mathcal{L}}_{c l}\right) t} q_{W}\right](\mathbf{x}, \mathbf{p})+\mathcal{O}\left(\hbar^{-f+1}\right) \\
& -\frac{\omega}{2 \pi \hbar} \int d \epsilon \partial_{\epsilon} p^{F D}(\epsilon) \sum_{p, r} \frac{\cos \left(\frac{r}{\hbar} S_{p}-r \frac{\pi}{2} \nu_{p}\right)}{\left|\operatorname{det}\left(\mathbf{m}_{p}^{r}-\mathbf{I}\right)\right|^{1 / 2}} \int d t e^{i \omega t} \oint_{p} d \tau q_{W}(\tau) q_{W}(\tau+t)+O\left(\hbar^{0}\right) .
\end{aligned}
$$

Assuming that $\hat{q}=\hat{q}^{\dagger}$ is a Hermitian operator, its Weyl-Wigner transform is a real function of $(\mathbf{x}, \mathbf{p})$. Along a periodic orbit, we have the Fourier series

$$
q_{W}(\tau)=\sum_{n=-\infty}^{+\infty} q_{p, n} \exp \left(i \frac{2 \pi n}{T_{p}} \tau\right)
$$

where $T_{p}$ is the classical period and $q_{p,-n}=q_{p, n}^{*}$. We obtain that

$$
\int d t e^{i \omega t} \oint_{p} d \tau q_{W}(\tau) q_{W}(\tau+t)=2 \pi T_{p}(\epsilon) \sum_{n=-\infty}^{+\infty}\left|q_{p, n}(\epsilon)\right|^{2} \delta\left[\omega-\frac{2 \pi n}{T_{p}(\epsilon)}\right] .
$$

Except in special systems like the harmonic oscillators, the periods $T_{p}(\epsilon)$ vary with the energy $\epsilon$. Because of the presence of the Dirac distribution, we can perform the last integral over $\epsilon$ by using 


$$
\delta\left[\omega-\frac{2 \pi n}{T_{p}(\epsilon)}\right]=\frac{T_{p}^{2}}{2 \pi n \partial_{\epsilon} T_{p}} \delta\left[\epsilon-\epsilon_{p, n}(\omega)\right]
$$

where $\epsilon=\epsilon_{p, n}(\omega)$ is the energy obtained by solving the implicit equation $T_{p}(\epsilon)=2 \pi n / \omega$. We here assumed that the periods vary monotonously with energy, otherwise we find a su of terms instead of a single term in the right-hand member of the previous formula. Noting that

$$
\frac{d S_{p, n}}{d \omega}=\frac{d}{d \omega} S_{p}\left[\epsilon_{p, n}(\omega)\right]=-\frac{T_{p}^{3}}{2 \pi n \partial_{\epsilon} T_{p}}
$$

we have that

$$
\int d t e^{i \omega t} \oint_{p} d \tau q_{W}(\tau) q_{W}(\tau+t)=2 \pi \sum_{n}\left|q_{p, n}(\epsilon)\right|^{2}\left|\frac{d S_{p, n}}{d \omega}\right| \delta\left[\epsilon-\epsilon_{p, n}(\omega)\right]
$$

where $S_{p, n}=S_{p}\left[\epsilon_{p, n}(\omega)\right]$.

Substituting in the previous relation, we finally obtain the absorptive part of the dynamical susceptibility as:

$$
\begin{aligned}
\tilde{\chi}^{\prime \prime}(\omega) & =-\frac{\omega}{2} \int d t \int \frac{d^{f} x d^{f} p}{(2 \pi \hbar)^{f}} \partial_{\epsilon} p^{F D}\left[h_{c l}(\mathbf{x}, \mathbf{p})\right] q_{W}(\mathbf{x}, \mathbf{p})\left[e^{\left(i \omega-\hat{\mathcal{L}}_{c l}\right) t} q_{W}\right](\mathbf{x}, \mathbf{p})+\mathcal{O}\left(\hbar^{-f+1}\right) \\
& -\left.\frac{\omega}{\hbar} \sum_{p, r, n} \partial_{\epsilon} p^{F D}\right|_{p, n}\left|q_{p, n}\right|^{2}\left|\frac{d S_{p, n}}{d \omega}\right| \frac{\cos \left(\frac{r}{\hbar} S_{p, n}-r \frac{\pi}{2} \nu_{p}\right)}{\left|\operatorname{det}\left(\mathbf{m}_{p, n}^{r}-\mathbf{I}\right)\right|^{1 / 2}}+O\left(\hbar^{0}\right)
\end{aligned}
$$

where all the expressions with indices $p, n$ are functions of the frequency $\omega$ through their dependency on the energy $\epsilon=\epsilon_{p, n}(\omega)$. Eq. (47) is the central result of this work.

To interpret this result, let us go back to the time correlation function $C_{\epsilon}(t)$ which is

$$
\operatorname{tr} \delta(\epsilon-\hat{h}) \exp (i \hat{h} t / \hbar) \hat{q} \exp (-i \hat{h} t / \hbar) \hat{q}=\frac{1}{2 \pi} \int d \tau e^{i \epsilon \tau / \hbar} \operatorname{tr} e^{i \hat{h}(t-\tau) / \hbar} \hat{q} e^{-i \hat{h} t / \hbar} \hat{q}
$$

In this expression, the trace can be rewritten in terms of the Green propagators as

$$
\begin{aligned}
& \operatorname{tr} \exp [i \hat{h}(t-\tau) / \hbar] \hat{q} \exp [-i \hat{h} t / \hbar] \hat{q} \\
= & \int d \mathbf{x} d \mathbf{x}^{\prime} G\left(\mathbf{x}^{\prime}, \mathbf{x} ; \tau-t\right) q(\mathbf{x}) G\left(\mathbf{x}, \mathbf{x}^{\prime} ; t\right) q\left(\mathbf{x}^{\prime}\right) \\
= & \sum_{c, c^{\prime}} \int d \mathbf{x} d \mathbf{x}^{\prime} A_{c}\left(\mathbf{x}^{\prime}, \mathbf{x} ; \tau-t\right) \exp \left[i W_{c}\left(\mathbf{x}^{\prime}, \mathbf{x} ; \tau-t\right) / \hbar\right] q(\mathbf{x}) \\
\times & A_{c^{\prime}}\left(\mathbf{x}, \mathbf{x}^{\prime} ; t\right) \exp \left[i W_{c^{\prime}}\left(\mathbf{x}, \mathbf{x}^{\prime} ; t\right) / \hbar\right] q\left(\mathbf{x}^{\prime}\right)
\end{aligned}
$$


where in the last step, we have written down symbolically the semiclassical expressions for the Green propagators. We have here assumed that the operator $\hat{q}$ depends only on the position operator, i.e., $\hat{q}=q(\hat{\mathbf{x}})$. The sum in the above expressions is over classical paths, $c$ and $c^{\prime}$. Notice that these are two different paths in general as they arise from two different propagators. The expression interprets for itself - from the point $\mathbf{x}$ to $\mathbf{x}^{\prime}$, we propagate along the path $c^{\prime}$ for time $t$, and then we propagate back to the point $\mathbf{x}$ along $c$ in time $\tau-t$. Precisely,

$$
\begin{aligned}
W_{c}\left(\mathbf{x}^{\prime} \rightarrow \mathbf{x} ; \tau-t\right) & =\int_{0}^{\tau-t} d t L \\
W_{c^{\prime}}\left(\mathbf{x} \rightarrow \mathbf{x}^{\prime} ; t\right) & =\int_{0}^{t} d t L,
\end{aligned}
$$

$L$ being the Lagrangian of the system. The total phase in the correlation function is the sum $W_{c}+W_{c^{\prime}}$ of the two actions. By stationary phase method, it can be shown that the two segments of the paths $c$ and $c^{\prime}$, in fact, form a periodic orbit of period $\tau$. This is the mechanism by which periodic orbit corrections appear in the main result above.

Classical contribution comes from the paths of zero length, i. e., $\tau=0$, and this is precisely the leading term in our expression, also called the quasiclassical term. Thus, in any calculation of interest, we need to find the classical correlation function, the Weyl corrections, and then the periodic-orbit corrections. If the energy spectrum of the system is discrete, we notice that the dynamical susceptibility (24) is a sum of Dirac peaks centered on each of the Bohr frequencies $\omega=\left(E_{m}-E_{n}\right) / \hbar$. In the semiclassical formula (47) the first term due to the paths of zero length contribute to the dynamical susceptibility by a continuous function of frequency $\omega$ which may be interpreted as the average or background of the discrete quantum susceptibility. The second series of terms of (47) are due to the periodic orbits which contribute by oscillatory functions of the frequency $\omega$. Their sum is supposed to reproduce the Dirac peaks of the discrete quantum susceptibility $\tilde{\chi} "(\omega)$.

We notice that, in contrast with the well-known Gutzwiller formula for the level density, the quasiclassical term here involves a classical time correlation function. As a consequence, the spectral properties of the classical Liouvillian $\hat{\mathcal{L}}_{c l}$ will intervene in the evaluation of this 
term (as well as of the following Weyl corrections). In this regard, we mention that a periodicorbit theory of the classical Liouvillian has been developed 17,18 in terms of classical zeta functions which resemble but differ from the Selberg-Gutzwiller quatum zeta function. Consequently, we have here an example where the semiclassical evaluation of a quantum property, namely $\tilde{\chi}^{\prime}(\omega)$, involves the spectral properties of the classical Liouvillian along with the Gutzwiller quantum periodic-orbit sum. It is remarkable that both these classical and semiclassical terms coexist within the same formula, which points to the result that the classical periodic-orbit theory is only one part of the underlying quantum dynamics. We believe that this result is general in the semiclassical evaluation of dynamical susceptibilities, which include in particular the ac conductivity.

It turns out that the spectral properties of the Liouvillian differ considerably whether the classical system $h_{c l}$ is integrable or chaotic.

For integrable systems, the Liouvillian spectrum is formed by discrete classical frequen-

cies, $\hat{\mathcal{L}}_{c l} \psi_{\mathbf{m}}=i \Omega_{\mathbf{m}} \psi_{\mathbf{m}}$, on each ergodic component which are the invariant tori. The expression would then be similar to the one obtained in [7]. The periodic-orbit corrections should be given by the Berry-Tabor semiclassical theory under such circumstances [22].

For chaotic systems, the classical time correlation function in the quasiclassical term decays if the system is mixing on the energy shell. Recent works have shown that this decay may be developed in terms of the complex singularities of an analogue of the Liouvillian resolvent 17, 18,23]. When the complex singularities are poles they are called Pollicott-Ruelle resonances and are associated with exponential decays. If the spectral decomposition of the Liouvillian dynamics in terms of the Pollicott-Ruelle resonances is known the quasiclassical term can be further reduced as a sum over the Pollicott-Ruelle resonances over each energy shell [17, 18,23].

\section{Concluding Remarks}

We have described a systematic procedure which lets us write important quantities in many-body theory in a semiclassical expansion. All our considerations are restricted to many-body systems reducible to uncoupled one-body systems, we believe that it is an inter- 
esting first step. We have shown the central role played by the time correlation functions. On the one hand, they are related by a Fourier transform to the response function; on the other hand, they facilitate useful expansions in terms of $\hbar$. The fact that the Fermionic nature of the particles has been taken into account is an important aspect. The other reason for an interest in the above considerations stems from the fact that no assumptions are made about integrability of the underlying classical one-body dynamics. It may be recalled that in a recent attempt along similar lines [7], complete integrability was assumed. Moreover, in their analysis, the Berry-Tabor formula for periodic orbit corrections has been used which fails for the interesting case of the harmonic oscillators. Since we now know that the Gutzwiller trace formula is exact for harmonic oscillators [2], and is also applicable for chaotic systems, we believe that our analysis extends to more general systems.

There are many applications of such a formalism. In any discussion of relaxation phenomena, time correlation functions play an important role. To understand photoabsorption cross-section of atoms and molecules, semiclassical treatment along these lines has been used 11.21. In metallic clusters and quantum dots, there is a growing interest in the semiclassical treatment of the absorption spectra and cross-sections. The understanding of the plasmon modes in different metallic clusters, their splitting etc. are believed to have a semiclassical interpretation. However, for a systematic treatment of the collective modes, we need to consider two-body interactions, thus a Hamiltonian,

$$
\hat{H}=\sum_{i} \hat{h}_{i}+\lambda \sum_{i j} \hat{v}_{i j}
$$

where $\hat{v}_{i j}$ denotes the two-body term. What we need to do here is a perturbative treatment of the two-body interaction by assuming that $\lambda \ll 1$ which, in turn, would give rise to collective modes.

In a random-matrix framework, one can also study the correlation functions, and it has been recently shown that the time correlations depend upon the co-dimension of level crossing [24]. A comprehensive understanding of all the inter-relations will be one of the aims we look forward to. 
Acknowledgements S.R.J. is financially supported by the "Communaute Francaise de Belgique" under contract no. ARC-93/98-166. S.R.J. is on an extraordinary leave from the Theoretical Physics Division, Bhabha Atomic Research Centre, Bombay 400 085, India. P. G. is grateful to the National Fund for Scientific Research (F. N. R. S. Belgium) for financial support. 


\section{REFERENCES}

[1] P. Gaspard, D. Alonso, and I. Burghardt, Adv. Chem. Phys. XC, 105 (1995).

[2] M. Brack and S. R. Jain, Phys. Rev. A 51, 3462 (1995).

[3] S. R. Jain and S. V. Lawande, Proc. Ind. Natl. Sc. Acad. 61 A, 275 (1995).

[4] P. van Ede van der Pals and P. Gaspard, Phys. Rev. E 49, 79 (1994).

[5] J. A. Folk et al., Phys. Rev. Lett. 76, 1699 (1996).

[6] M. Brack, Rev. Mod. Phys. 65, 677(1993).

[7] A. Dellafiore, F. Matera, and D. M. Brink, Phys. Rev. A 51, 914 (1995).

[8] S. R. Jain, A. K. Jain, and Z. Ahmed, Phys. Lett. B 370, 1 (1996).

[9] D. M. Brink, A. Dellafiore, and M. Di Toro, Nucl. Phys. A 456, 205 (1986).

[10] H. Nishioka, K. Hansen, and B. R. Mottelson, Phys. Rev. B 42, 9377 (1990).

[11] V. Strutinsky, A. G. Magner, S. R. Ofengenden, and T. Dossing, Z. Phys. A 283, 269 (1977).

[12] M. C. Gutzwiller, Chaos in Classical and Quantum Mechanics (Springer, New York, 1991).

[13] R. Balescu, Equilibrium and Nonequilibrium Statistical Mechanics (John Wiley and Sons, Inc., New York, 1974).

[14] A. L. Fetter and J. D. Walecka, Quantum Theory of Many-Particle Systems (McGraw Hill Inc., New York, 1971).

[15] B. Eckardt, S. Fishman, K. Müller, and D. Wintgen, Phys. Rev. A 45, 3531 (1992).

[16] This famous trick is first known to have been applied by W. Pauli (Cf. Ref. [14]). The relevant reference for the present context is the paper by P. Gaspard and S. A. Rice, 
Phys. Rev. A 48, 54 (1993).

[17] R. Artuso, E. Aurell, and P. Cvitanović, Nonlinearity 3, 325, 361 (1990); P. Cvitanović and B. Eckhardt, J. Phys. A: Math. Gen. 24, L237 (1991).

[18] P. Gaspard and D. Alonso Ramirez, Phys. Rev. A 45, 8383 (1992).

[19] M. L. Du and J. B. Delos, Phys. Rev. A 38, 1896, 1913 (1988).

[20] N. L. Balasz and B. K. Jennings, Phys. Rep. 104, 347 (1984).

[21] D. Alonso Ramirez, Semiclassical Quantization and Classically Chaotic Systems, Ph. D. Thesis (Université Libre de Bruxelles, unpublished, 1995).

[22] M.V. Berry and M. Tabor, Proc. R. Soc. Lond A. 349, 101-123, (1976); M. V. Berry and M. Tabor, J. Phys. A: Math. Gen. 10, 371 (1977).

[23] M. Pollicott, Invent. Math. 81, 413 (1985); D. Ruelle, Phys. Rev. Lett. 56, 405 (1986); J. Stat. Phys. 44, 281 (1986); J. Diff. Geom. 25, 99, 117 (1987).

[24] P. Gaspard, S. R. Jain, and P. van Ede van der Pals, Time Correlation Functions of Complex Quantum Systems (preprint, 1996). 